\title{
Weighted Maximum Variance Dimensionality Reduction
}

\author{
Turki Turki ${ }^{1,2}$ and Usman Roshan ${ }^{2}$ \\ 1 Computer Science Department, King Abdulaziz University \\ P.O. Box 80221, Jeddah 21589, Saudi Arabia \\ tturki@kau.edu.sa \\ 2 Department of Computer Science, New Jersey Institute of Technology \\ University Heights, Newark, NJ 07102 \\ usman@cs.njit.edu
}

\begin{abstract}
Dimensionality reduction procedures such as principal component analysis and the maximum margin criterion discriminant are special cases of a weighted maximum variance (WMV) approach. We present a simple two parameter version of WMV that we call $2 \mathrm{P}-\mathrm{WMV}$. We study the classification error given by the 1-nearest neighbor algorithm on features extracted by our and other dimensionality reduction methods on several real datasets. Our results show that our method yields the lowest average error across the datasets with statistical significance.
\end{abstract}

Keywords: dimensionality reduction, principal component analysis, maximum margin criterion.

\section{Introduction}

The problem of dimensionality reduction arises in many data mining and machine learning tasks. Among many such algorithms the principal component analysis [1] (PCA) is a very popular choice. PCA seeks a vector $w \in R^{d}$ that solves

$$
\arg \max _{w} \frac{1}{2 n} \sum_{i, j} \frac{1}{n}\left(w^{T}\left(x_{i}-x_{j}\right)\right)^{2}
$$

where $x_{i} \in R^{d}$ for $i=0 \ldots n-1$. In other words it maximizes the variance of the projected data without taking class labels into consideration. The maximum margin criterion (MMC) 2] is a supervised dimensionality reduction method that overcomes limitations of the Fisher linear discriminant and has also shown to achieve higher classification accuracy [2. It is given by $w$ that maximizes trace $\left(w^{T}\left(S_{b}-S_{w}\right) w\right)$ subject to $w^{T} w=I$. Using Lagrange multipliers one can show that $w$ is given by the largest eigenvectors of $S_{b}-S_{w}$.

In this paper we consider a general version of Equation 1 that we call the maximum weighted variance given by

$$
\arg \max _{w} \frac{1}{2 n} \sum_{i, j} C_{i j}\left(w^{T}\left(x_{i}-x_{j}\right)\right)^{2}
$$


The above equation gives us both PCA and MMC for specific settings of $C_{i j}$ as we show below. We consider a two parameter approach by setting $C_{i j}=\alpha<0$ if $x_{i}$ and $x_{j}$ have the same class label and $C_{i j}=\beta>0$ otherwise. In other words we simultaneously minimize the distance between projected pairwise points in the same class and maximize the same distance for points in different classes. For a given dataset we obtain $\alpha$ and $\beta$ by 1-nearest neighbor cross-validation.

The straightforward eigendecomposition solution requires at least quadratic space in the dimensions of $x_{i}$. With graph Laplacians we can employ a singular value decomposition (SVD) approach to avoid this problem (as originally given in [3]) and thus apply it to high dimensional data. Below we describe our approach in detail followed by experimental results.

\section{Methods}

Suppose we are given the vectors $x_{i} \in R^{d}$ for $i=0 \ldots n-1$ and a real matrix $C \in R^{n \times n}$. Let $X$ be the matrix containing $x_{i}$ as its columns (ordered $x_{0}$ through $\left.x_{n-1}\right)$. Now consider the optimization problem

$$
\arg \max _{w} \frac{1}{2 n} \sum_{i, j} C_{i j}\left(w^{T}\left(x_{i}-x_{j}\right)\right)^{2}
$$

where $w \in R^{d}$ and $C_{i j}$ is the entry in $C$ corresponding to the $i^{\text {th }}$ row and $j^{\text {th }}$ column. This is in fact a more general representation of PCA and MMC.

\subsection{Principal Component Analysis}

To obtain PCA we set $C_{i j}=\frac{1}{n}$ and Equation 3 becomes (without the arg $\max$ part)

$$
\begin{aligned}
& \frac{1}{2 n} \sum_{i, j} \frac{1}{n}\left(w^{T}\left(x_{i}-x_{j}\right)\right)^{2}= \\
& \frac{1}{2 n} \sum_{i, j} \frac{1}{n} w^{T}\left(x_{i}-x_{j}\right)\left(x_{i}-x_{j}\right)^{T} w= \\
& \frac{1}{2 n} \sum_{i, j} \frac{1}{n} w^{T}\left(x_{i} x_{i}^{T}-x_{i} x_{j}^{T}-x_{j} x_{i}^{T}+x_{j} x_{j}^{T}\right) w= \\
& \frac{1}{2 n} w^{T} \frac{1}{n}\left(\sum_{i, j}\left(x_{i} x_{i}^{T}-x_{i} x_{j}^{T}-x_{j} x_{i}^{T}+x_{j} x_{j}^{T}\right)\right) w= \\
& \frac{1}{2 n} w^{T} \frac{1}{n}\left(2 \sum_{i, j} x_{i} x_{i}^{T}-2 \sum_{i, j} x_{i} x_{j}^{T}\right) w= \\
& \frac{1}{2 n} w^{T} \frac{1}{n}\left(2 n \sum_{i} x_{i} x_{i}^{T}-2 n^{2} m m^{T}\right) w= \\
& \frac{1}{n} w^{T}\left(\sum_{i} x_{i} x_{i}^{T}-n m m^{T}\right) w= \\
& w^{T}\left(\frac{1}{n} \sum_{i}\left(x_{i}-m\right)\left(x_{i}-m\right)^{T}\right) w= \\
& w^{T} S_{t} w
\end{aligned}
$$


where $S_{t}=\frac{1}{n} \sum_{i}\left(x_{i}-m\right)\left(x_{i}-m\right)^{T}$ and is called the total scatter matrix. Inserting the optimization criterion into the last step yields $\arg \max _{w} w^{T} S_{t} w$ which is exactly the PCA optmization criterion 1 .

\subsection{Maximum Margin Discriminant}

To obtain the MMC discriminant (a supervised learning method) first recall that the MMC optimization criterion is defined as $\arg \max _{w} w^{T}\left(S_{b}-S_{w}\right) w$ where $S_{b}$ is the between-class scatter matrix and $S_{w}$ is the within-class scatter matrix [2]. Since $S_{b}-S_{w}=S_{t}-2 S_{w}$ where $S_{t}$ is the total scatter matrix, this can be written as $\arg \max _{w} w^{T}\left(S_{t}-2 S_{w}\right) w$ [4. In practice though we would use the weighted maximum margin discriminant which is given by $\arg \max _{w} w^{T}\left(S_{b}-\alpha S_{w}\right) w[5$. We now set the weights $C_{i j}$ to obtain this discriminant.

Suppose class labels $y_{i} \in\{+1,-1\}$ are provided for each $x_{i}$ and $n_{k}$ is the size of class $k$. Define $C_{i j}$ to be $\frac{1}{n}$ if $i$ and $j$ have different class labels and $\frac{1}{n}-2 \frac{1}{n_{k}}$ if $i$ and $j$ have the same class labels. We can then write Equation 3 as

$$
\arg \max _{w} \frac{1}{2 n}\left(\sum_{i, j} G_{i j}\left(w^{T}\left(x_{i}-x_{j}\right)\right)^{2}-\sum_{i, j} 2 L_{i j}\left(w^{T}\left(x_{i}-x_{j}\right)\right)^{2}\right)
$$

where $G_{i j}=\frac{1}{n}$ for all $i$ and $j$ and $L_{i j}=\frac{1}{n_{k}}$ if $i$ and $j$ have class labels $k$ and 0 otherwise. By substituting the values of $G_{i j}$ and $L_{i j}$ into Equation 4 and some symbolic manipulation we obtain the MMC discriminant

$$
\begin{aligned}
& \frac{1}{2 n} \sum_{i, j} w^{T}\left(G_{i j}\left(x_{i}-x_{j}\right)\left(x_{i}-x_{j}\right)-2 L_{i j}\left(x_{i}-x_{j}\right)\left(x_{i}-x_{j}\right)^{T}\right) w= \\
& \frac{1}{2 n}\left(\sum_{i, j} \frac{1}{n} w^{T}\left(x_{i}-x_{j}\right)\left(x_{i}-x_{j}\right)^{T} w-\right. \\
& \left.2 \sum_{k=1}^{c} \sum_{c l\left(x_{j}\right)=k, c l\left(x_{i}\right)=k} \frac{1}{n_{k}} w^{T}\left(x_{i}-x_{j}\right)\left(x_{i}-x_{j}\right)^{T} w\right)= \\
& \frac{1}{2 n}\left(2 \sum_{i}^{n} w^{T}\left(x_{i}-m\right)\left(x_{i}-m\right) w-\right. \\
& \left.2 \sum_{k=1}^{c} \frac{1}{n_{k}} \sum_{c l\left(x_{j}\right)=k, c l\left(x_{i}\right)=k} w^{T}\left(x_{i} x_{i}^{T}-x_{i} x_{j}^{T}-x_{j} x_{i}^{T}+x_{j} x_{j}^{T}\right) w\right)= \\
& \frac{1}{2 n}\left(2 \sum_{i}^{n} w^{T}\left(x_{i}-m\right)\left(x_{i}-m\right) w-\right. \\
& \left.2 \sum_{k=1}^{c} \frac{1}{n_{k}} \sum_{c l\left(x_{j}\right)=k, c l\left(x_{i}\right)=k} w^{T}\left(2 x_{i} x_{i}^{T}-2 x_{i} x_{j}^{T}\right) w\right)= \\
& \frac{1}{2 n}\left(2 \sum_{i}^{n} w^{T}\left(x_{i}-m\right)\left(x_{i}-m\right) w-\right. \\
& \left.2 \sum_{k=1}^{c} \frac{1}{n_{k}} \sum_{c l\left(x_{i}\right)=k} w^{T}\left(2 n_{k} x_{i} x_{i}^{T}-2 n_{k}^{2} m_{k} m_{k}^{T}\right) w\right)= \\
& \frac{1}{n}\left(\sum_{i}^{n} w^{T}\left(x_{i}-m\right)\left(x_{i}-m\right) w-\right. \\
& 2 \sum_{k=1}^{c} \sum_{c l}\left(x_{i}\right)=k \\
& \left.w^{T}\left(x_{i} x_{i}^{T}-n_{k} m_{k} m_{k}^{T}\right) w\right)= \\
& \frac{1}{n}\left(\sum_{i}^{n} w^{T}\left(x_{i}-m\right)\left(x_{i}-m\right) w-\right. \\
& \left.\left.2 \sum_{k=1}^{c} \sum_{c l\left(x_{i}\right)=k} w^{T}\left(x_{i}-m_{k}\right)\left(x_{i}-m_{k}\right)^{T}\right) w\right)= \\
& w^{T}\left(S_{t}-2 S_{w}\right) w
\end{aligned}
$$


where $m_{k}$ is the mean of points in $k$ and $c l(x)$ returns the class of point $x$. The last equation in the above steps is just the MMC discriminant.

Equation 3 can be rewritten as $\arg \max _{w} \frac{1}{n} w^{T} X L X^{T} w$ where $L=D-C$ and $D_{i i}=\sum_{i} C_{i i}[$ ]. The matrix $L$ is called the Laplacian of the weight matrix $C$. Using Lagrange multipliers one can show that the largest eigenvector of $\frac{1}{n} X L X^{T}$ (i.e. eigenvector with largest eigenvalue) is the solution to $w[6]$. Thus, the largest eigenvector is also the solution to PCA and MMC.

\subsection{Laplacian Linear Discriminant Analysis}

Following the Laplacian framework we can write the MMC discriminant (Equation 4) as arg $\max _{w} \frac{1}{n} w^{T} X\left(L_{g}-2 L_{l}\right) X^{T} w$ where $L_{g}$ is the Laplacian of $G$ and $L_{l}$ is the Laplacian of $L$ 3.4. This form of the the maximum margin discriminant is also called Laplacian linear discriminant analysis and has been studied for unsupervlsed learning [4. As in PCA and MMC the largest eigenvector of $\frac{1}{n} X\left(L_{g}-2 L_{l}\right) X^{T}$ is the solution to the Laplacian discriminant.

Notice that $C_{i j}$ in Equation 3 can take on arbitrary values. With suitable settings we obtained PCA and MMC. How does one select the best values $C_{i j}$ for a particular problem? Our solution is to collapse values of $C$ into two parameters and select their values that minimize error on the training data.

\subsection{Two Parameter Weighted Maximum Variance Discriminant}

As shown above the MMC discriminant is obtained by setting $G_{i j}=\frac{1}{n}$ for all $i$ and $j$ and $L_{i j}=\frac{1}{n_{k}}$ if $i$ and $j$ have class labels $k$ and 0 otherwise in Equation 4 . We consider a different setting for $L$ below which gives us the two parameter weighted maximum variance discriminant (2P-WMV). We also show that this yields a class-wise unnormalized within-class scatter matrix and a pairwise interclass scatter matrix.

Define the matrix $G \in R^{n \times n}$ as $G_{i j}=\frac{1}{n}$ for all $i$ and $j$ and $L \in R^{n \times n}$ as

$$
L_{i j}= \begin{cases}\alpha & \text { if } y_{i}=y_{j} \\ \beta & \text { if } y_{i} \neq y_{j} \\ 0 \text { if } y_{i} \text { or } y_{j} \text { is undefined }\end{cases}
$$

Substituting these values into Equation 4 we obtain 


$$
\begin{aligned}
& \frac{1}{2 n}\left(\sum_{i, j} \frac{1}{n} w^{T}\left(x_{i}-x_{j}\right)\left(x_{i}-x_{j}\right) w\right. \\
& -2 \sum_{c l\left(x_{i}\right)=c l\left(x_{j}\right)} \alpha w^{T}\left(x_{i}-x_{j}\right)\left(x_{i}-x_{j}\right)^{T} w \\
& \left.-2 \sum_{c l\left(x_{i}\right) \neq c l\left(x_{j}\right)} \beta w^{T}\left(x_{i}-x_{j}\right)\left(x_{i}-x_{j}\right)^{T} w\right)= \\
& \frac{1}{2 n}\left(2 \sum_{i}^{n} w^{T}\left(x_{i}-m\right)\left(x_{i}-m\right) w\right. \\
& -2 \sum_{k=1}^{c} \alpha 2 n_{k} \sum_{c l\left(x_{j}\right)=k} w^{T}\left(x_{j}-m_{k}\right)\left(x_{j}-m_{k}\right)^{T} w \\
& \left.-2 \beta \sum_{c=1}^{k} \sum_{d=c+1}^{k} \sum_{c l\left(x_{i}\right)=c, c l\left(x_{j}\right)=d} w^{T}\left(x_{i}-x_{j}\right)\left(x_{i}-x_{j}\right)^{T} w\right)= \\
& \frac{1}{n} \sum_{i}^{n} w^{T}\left(x_{i}-m\right)\left(x_{i}-m\right) w \\
& -2 \alpha \frac{1}{n} \sum_{k=1}^{c} n_{k} \sum_{c l\left(x_{j}\right)=k} w^{T}\left(x_{j}-m_{k}\right)\left(x_{j}-m_{k}\right)^{T} w \\
& -2 \beta \frac{1}{n} \sum_{c=1}^{k} \sum_{d=c+1}^{k} \sum_{c l\left(x_{i}\right)=c, c l\left(x_{j}\right)=d} w^{T}\left(x_{i}-x_{j}\right)\left(x_{i}-x_{j}\right)^{T} w= \\
& w^{T} S_{t} w-2\left(\alpha w^{T} S_{w}^{\prime} w+\beta w^{T} S_{b}^{\prime} w\right)= \\
& w^{T}\left(S_{t}-2\left(\alpha S_{w}^{\prime}+\beta S_{b}^{\prime}\right)\right) w
\end{aligned}
$$

where

$$
\begin{aligned}
& S_{w}^{\prime}=\frac{1}{n} \sum_{k=1}^{c} n_{k} \sum_{c l\left(x_{j}\right)=k}\left(x_{j}-m_{k}\right)\left(x_{j}-m_{k}\right)^{T} \\
& S_{b}^{\prime}=\frac{1}{2 n} \sum_{c=1}^{k} \sum_{d=c+1}^{k} \sum_{c l\left(x_{i}\right)=c, c l\left(x_{j}\right)=d}\left(x_{i}-x_{j}\right)\left(x_{i}-x_{j}\right)^{T}
\end{aligned}
$$

Note the similarity of $S_{w}^{\prime}$ to the standard within-class matrix used in MMC given by $S_{w}=\frac{1}{n} \sum_{i}^{k} \sum_{c l\left(x_{j}\right)=i}\left(x_{j}-m^{i}\right)\left(x_{j}-m^{i}\right)^{T} . S_{w}$ is the class-wise normalized version of $S_{w}^{\prime}$. Thus, the discriminant yielded by our approach is given by the standard total scatter matrix, a modified within-class matrix, and a pairwise inter-class scatter matrix. We can obtain MMC by setting $\alpha=\frac{1}{n_{k}}$ if $y_{i}=k, y_{j}=k$ and $\beta=0$. This discards the inter-class scatter matrix and makes $S_{w}^{\prime}=S_{w}$.

After defining $L$ and $G$ compute $L_{g}$ the Laplacian of $G, L_{l}$ the Laplacian of $L$, and the matrix $\frac{1}{n} X\left(L_{g}-L_{l}\right) X^{T}$ (the 2P-WMV discriminant). The solution to $2 \mathrm{P}-\mathrm{WMV}$ is $w$ that maximizes $\frac{1}{n} w^{T} X\left(L_{g}-L_{l}\right) X^{T} w$ which is in turn is given by the largest eigenvector of $\frac{1}{n} X\left(\stackrel{n}{L}_{g}-L_{l}\right) X^{T}$ [4].

\section{Results}

To evaluate the classification ability of our extracted features we use the simple and popular 1-nearest neighbor (1NN) algorithm. In 10-fold and 5-fold crossvalidation experiments we apply the 1-nearest neighbor classification algorithm to features extracted from our method 2P-WMV, the weighted maximum margin discriminant (WMMC), PCA, and the features as they are (denoted simply as $1 \mathrm{NN})$. We calculate average error rates across 50 randomly selected datasets shown in Table 1 from the UCI Machine Learning Repository [7. 
Table 1. Datasets from the UCI Machine Learning repository that we used in our study 7

\begin{tabular}{|c|c|c|c|c|}
\hline \multicolumn{2}{|c|}{ Code Dataset } & \multicolumn{3}{|c|}{ Classes Dimension Instances } \\
\hline 1 & Liver-disorders & 2 & 6 & 345 \\
\hline 2 & Diabetes & 2 & 8 & 768 \\
\hline 3 & Breast Cancer & 2 & 10 & 683 \\
\hline 4 & Page block & 5 & 10 & 5473 \\
\hline 5 & Wine-quality-red & 11 & 11 & 1599 \\
\hline 6 & Wine quality & 11 & 11 & 4898 \\
\hline 7 & Wine & 3 & 13 & 178 \\
\hline 8 & Heart & 2 & 13 & 270 \\
\hline 9 & Australian Credit Approval & 2 & 14 & 690 \\
\hline 10 & EEG Eye State & 2 & 14 & 14980 \\
\hline 11 & Pen-Based Recognition & 10 & 16 & 10992 \\
\hline 12 & Climate & 2 & 18 & 540 \\
\hline 13 & lymphography & 4 & 18 & 148 \\
\hline 14 & Statlog image & 7 & 19 & 2310 \\
\hline 15 & Two norm & 2 & 20 & 7400 \\
\hline 16 & Ring & 2 & 20 & 7400 \\
\hline 17 & Cardiotocography & 10 & 21 & 2126 \\
\hline 18 & Thyroid & 3 & 21 & 7200 \\
\hline 19 & Waveform & 3 & 21 & 5000 \\
\hline 20 & Statlog German credit card & 2 & 24 & 1000 \\
\hline 21 & Steel faults & 7 & 27 & 1941 \\
\hline 22 & Breast cancer & 2 & 30 & 569 \\
\hline 23 & Ionosphere & 2 & 34 & 351 \\
\hline 24 & Dermatology & 6 & 34 & 366 \\
\hline 25 & Statlog & 7 & 36 & 6435 \\
\hline 26 & Texture & 11 & 40 & 5500 \\
\hline 27 & Waveform & 3 & 40 & 5000 \\
\hline 28 & Qsar & 2 & 41 & 1055 \\
\hline 29 & SPECTF heart & 2 & 44 & 267 \\
\hline 30 & Mlprove & 6 & 51 & 6118 \\
\hline 31 & Spambase & 2 & 57 & 4597 \\
\hline 32 & Sonar & 2 & 60 & 208 \\
\hline 33 & Digits & 2 & 63 & 762 \\
\hline 34 & Ozone & 2 & 72 & 1847 \\
\hline 35 & Insurance company coil2000 & 2 & 85 & 5822 \\
\hline 36 & Movement libras & 15 & 90 & 360 \\
\hline 37 & Hill valley & 2 & 100 & 606 \\
\hline 38 & $\mathrm{BCI}$ & 2 & 117 & 400 \\
\hline 39 & Gas sensor array drift & 6 & 128 & 13910 \\
\hline 40 & Musk & 2 & 166 & 476 \\
\hline 41 & Coil & 6 & 241 & 1500 \\
\hline 42 & Scene classification & 6 & 294 & 2230 \\
\hline 43 & Madelon & 2 & 500 & 2600 \\
\hline 44 & Smartphone & 6 & 561 & 10299 \\
\hline 45 & Secom & 2 & 591 & 1567 \\
\hline 46 & Mfeat & 10 & 649 & 2000 \\
\hline 47 & CNAE-9 & 9 & 857 & 1080 \\
\hline 48 & ACASVA actions & 2 & 960 & 11288 \\
\hline 49 & Micromass & 2 & 1300 & 931 \\
\hline 50 & Gisette & 2 & 5000 & 1000 \\
\hline
\end{tabular}




\subsection{Experimental Methodology}

We compare four classification algorithms: $2 \mathrm{P}-\mathrm{WMV}+1 \mathrm{NN}, \mathrm{PCA}+1 \mathrm{NN}$, $\mathrm{WMMC}+1 \mathrm{NN}$, and $1 \mathrm{NN}$ where the first three are $1 \mathrm{NN}$ applied to features extracted from each of the three dimensionality reduction algorithms. We use 10-fold cross-validation on each real dataset with the same set of splits for each algorithm. However, for datasets with fewer than 300 instances we use 5 -fold crossvalidation to obtain a large enough validation set. For dimensionality reduction we find the best parameters and number of dimensions by cross-validating further on the training dataset (also 10-fold).

In $2 \mathrm{P}-\mathrm{WMV}$ we let $\beta$ range from $\{-2,-1.9,-1.8,-1.7,-1.6,-1.5,-1.4,-1.3,-1.2,-1.1,-$ $1,-.9,-.8,-. .7,-.6,-.5,-.4,-. .3,-.2,-.1,-.01\}$ and $\alpha$ fixed to 1 . For WMMC we let the $\alpha$ parameter range from $\left\{10^{-7}, 10^{-6}, 10^{-5}, 10^{-4}, 10^{-3}, 10^{-2}, 10^{-1}, 1,10,100\right\}$. Recall that WMMC is given by $\arg \max _{w} w^{T}\left(S_{b}-\alpha S_{w}\right) w[5]$. For each parameter we reduce dimensionality to 20 and then pick the top $1<=k<=20$ features that give the lowest $1 \mathrm{NN}$ error on the training. Thus the cross-validation on the training set gives us the best values of $\alpha$ and the reduced number of features (including PCA) which we then apply to the validation set.

We wrote our code in $\mathrm{C}$ and $\mathrm{R}$ and make it freely available at http://www.cs.njit.edu/usman/wmv/. Our C programs use CLAPACK libraries for performing the eigenvector and singular value decompositions.

\subsection{Experimental Results on Fifty Datasets}

We compute the balanced error rate [8] for each training-validation split during cross-validation and take the mean to be the average cross-validation error. In Table 2 we show the average cross-validation error on each dataset. Across the the 50 datasets $2 \mathrm{P}-\mathrm{WMV}+1 \mathrm{NN}$ achieves the lowest average error of $13.324 \%$ and has the lowest error in 21 out of the 50 datasets. The next best is WMMC+1NN that achieves an average error of $15.302 \%$ and has the lowest error in 12 out of the 50 datasets. $\mathrm{PCA}+1 \mathrm{NN}$ are $1 \mathrm{NN}$ have higher average errors at $18.765 \%$ and $18.946 \%$ respectively. $\mathrm{PCA}+1 \mathrm{NN}$ and $1 \mathrm{NN}$ have the lowest error in 2 and 9 out of the 50 datasets respectively.

We measure the statistical significance with the Wilcoxon rank test 9 . This is a standard test to measure the difference between two methods across a number of datasets. Roughly speaking it shows statistical significance between two methods when one outperforms the other each time on a large number of datasets. In Table 3 the p-values show that $2 \mathrm{P}-\mathrm{WMV}+1 \mathrm{NN}$ statistically significantly outperforms the other three method across all 50 datasets. 
Table 2. Average cross-validation error of different algorithms on each of the 50 real datasets from the UCI machine learning repository. Shown in bold is the method with the lowest unique error.

\begin{tabular}{|c|c|c|c|c|c|}
\hline \multicolumn{2}{|c|}{ Code Dataset } & \multicolumn{4}{|c|}{$2 \mathrm{P}-\mathrm{WMV}+1 \mathrm{NN}$ WMMC+1NN PCA+1NN $1 \mathrm{NN}$} \\
\hline 1 & Liver-disorders & 0.364 & 0.376 & 0.4 & 0.404 \\
\hline 2 & Diabetes & 0.31912 & 0.33382 & 0.34706 & 0.31912 \\
\hline 3 & Breast Cancer & 0.03016 & 0.03492 & 0.37937 & 0.37937 \\
\hline 4 & Page block & 0.04586 & 0.04199 & 0.04622 & 0.04622 \\
\hline 5 & Wine-quality-red & 0.37718 & 0.37785 & 0.42081 & 0.42013 \\
\hline 6 & Wine quality & 0.37582 & 0.38381 & 0.4043 & 0.40451 \\
\hline 7 & Wine & 0.075 & 0.075 & 0.2125 & 0.2125 \\
\hline 8 & Heart & 0.21 & 0.33 & 0.425 & 0.42 \\
\hline 9 & Australian Credit Approval & 0.20833 & 0.21667 & 0.44167 & 0.43167 \\
\hline 10 & EEG Eye State & 0.0198 & 0.02094 & 0.0202 & 0.0202 \\
\hline 11 & Pen-Based Recognition & 0.00586 & 0.00614 & 0.00577 & 0.00577 \\
\hline 12 & Climate & 0.066 & 0.072 & 0.14 & 0.132 \\
\hline 13 & Lymphography & 0.2 & 0.21786 & 0.21429 & 0.2 \\
\hline 14 & Statlog image & 0.03609 & 0.03435 & 0.03609 & 0.03565 \\
\hline 15 & Two norm & 0.0289 & 0.02918 & 0.03342 & 0.05315 \\
\hline 16 & Ring & 0.14685 & 0.14014 & 0.15425 & 0.24274 \\
\hline 17 & Cardiotocography & 0.08398 & 0.08932 & 0.08495 & 0.0835 \\
\hline 18 & Thyroid & 0.03915 & 0.06211 & 0.07014 & 0.07014 \\
\hline 19 & Waveform & 0.18143 & 0.18 & 0.18612 & 0.22857 \\
\hline 20 & Statlog German credit card & 0.33444 & 0.37 & 0.35667 & 0.35444 \\
\hline 21 & Steel faults & 0.36126 & 0.36073 & 0.61885 & 0.61885 \\
\hline 22 & Breast cancer & 0.07755 & 0.11429 & 0.09388 & 0.09388 \\
\hline 23 & Ionosphere & 0.06452 & 0.05806 & 0.10323 & 0.10968 \\
\hline 24 & Dermatology & 0.01538 & 0.03462 & 0.11538 & 0.11538 \\
\hline 25 & Statlog & 0.09118 & 0.11874 & 0.09496 & 0.09512 \\
\hline 26 & Texture & 0.00926 & 0.01315 & 0.00944 & 0.00796 \\
\hline 27 & Waveform & 0.18143 & 0.18755 & 0.17898 & 0.23837 \\
\hline 28 & Qsar & 0.18211 & 0.16211 & 0.20316 & 0.19895 \\
\hline 29 & SPECTF heart & 0.27647 & 0.25882 & 0.28235 & 0.26471 \\
\hline 30 & Mlprove & 0.42204 & 0.44128 & 0.41941 & 0.41382 \\
\hline 31 & Spambase & 0.08709 & 0.08249 & 0.17221 & 0.16565 \\
\hline 32 & Sonar & 0.17222 & 0.2 & 0.15556 & 0.15556 \\
\hline 33 & Digits & 0.01111 & 0.01806 & 0.01111 & 0.00972 \\
\hline 34 & Ozone & 0.10904 & 0.09718 & 0.10678 & 0.10565 \\
\hline 35 & Insurance company coil2000 & 0.1042 & 0.10262 & 0.0965 & 0.09685 \\
\hline 36 & Movement libras & 0.10333 & 0.12333 & 0.10333 & 0.09667 \\
\hline 37 & Hill valley & 0.02321 & 0.06429 & 0.41607 & 0.42143 \\
\hline 38 & $\mathrm{BCI}$ & 0.16333 & 0.17667 & 0.44667 & 0.41333 \\
\hline 39 & Gas sensor array drift & 0.00878 & 0.01058 & 0.00878 & 0.00885 \\
\hline 40 & Musk & 0.11957 & 0.23696 & 0.13478 & 0.1587 \\
\hline 41 & Coil & 0.02286 & 0.03429 & 0.02143 & 0.01429 \\
\hline 42 & Scene classification & 0.29454 & 0.335 & 0.29636 & 0.28909 \\
\hline 43 & Madelon & 0.1256 & 0.4568 & 0.1268 & 0.3444 \\
\hline 44 & Smartphone & 0.04563 & 0.04194 & 0.07363 & 0.02623 \\
\hline 45 & Secom & 0.08027 & 0.11429 & 0.1 & 0.10204 \\
\hline 46 & Mfeat & 0.05526 & 0.05158 & 0.05211 & 0.05263 \\
\hline 47 & CNAE-9 & 0.069 & 0.065 & 0.176 & 0.132 \\
\hline 48 & ACASVA actions & 0.11637 & 0.18479 & 0.17809 & 0.1178 \\
\hline 49 & Micromass & 0.07253 & 0.06264 & 0.11209 & 0.05934 \\
\hline \multirow[t]{2}{*}{50} & Gisette & 0.04889 & 0.05111 & 0.09556 & 0.08222 \\
\hline & Average error & 0.13324 & 0.15302 & 0.18765 & 0.18946 \\
\hline
\end{tabular}


Table 3. Wilcox rank test p-values (two-tailed test) between all pairs of methods

\begin{tabular}{cccc}
\hline & WMMC+1NN $P C A+1 \mathrm{NN}$ & $1 \mathrm{NN}$ \\
$2 \mathrm{P}-\mathrm{WMV}+1 \mathrm{NN}$ & .0004 & $<.0001$ & .0001 \\
WMMC+1NN & & .0232 & .0536 \\
$\mathrm{PCA}+1 \mathrm{NN}$ & & & .0949 \\
\hline
\end{tabular}

\section{Discussion}

Both $2 \mathrm{PWMV}+1 \mathrm{NN}$ and $\mathrm{WMMC}+1 \mathrm{NN}$ reduce dimensionality by determining optimal parameters specific to the given dataset. This approach is better than the unsupervised PCA and the non-parametric MMC (results not shown here). In fact $1 \mathrm{NN}$ applied to the raw data can be better than non-parameteric MMC most of the time.

In this study we fixed $\alpha$ for 2PWMV and varied only $\beta$. If we cross-validated $\alpha$ we could potentially obtain lower error but at the cost of increased running time. In the current experiments $2 \mathrm{PWMV}+1 \mathrm{NN}$ and $\mathrm{WMMC}+1 \mathrm{NN}$ are the slowest methods yet still tractable for large datasets.

We chose $1 \mathrm{NN}$ as the classification method for this study due to its simplicity and its popularity with dimensionality reduction programs. Other classifiers such as the support vector machine [1 may perform better when replaced with $1 \mathrm{NN}$. However, in that case the regularization parameter would also need to be optimized via cross-validation which increases the total runtime.

\section{Conclusion}

We introduce a two parameter variant of the weighted maximum variance discriminant and optimize it with cross-validation followed by 1-nearest neighbor for classification. Compared to existing approaches our method obtains the lowest average error with statistical significance across several real datasets from the UCI machine learning repository.

\section{References}

1. Alpaydin, E.: Machine Learning. MIT Press (2004)

2. Li, H., Jiang, T., Zhang, K.: Efficient and robust feature extraction by maximum margin criterion. In: Thrun, S., Saul, L., Schölkopf, B. (eds.) Advances in Neural Information Processing Systems 16. MIT Press, Cambridge (2004)

3. Tang, H., Fang, T., Shi, P.F.: Rapid and brief communication: Laplacian linear discriminant analysis. Pattern Recogn. 39(1), 136-139 (2006)

4. Niijima, S., Okuno, Y.: Laplacian linear discriminant analysis approach to unsupervised feature selection. IEEE/ACM Transactions on Computational Biology and Bioinformatics 6(4), 605-614 (2009)

5. Zheng, W., Zou, C., Zhao, L.: Weighted maximum margin discriminant analysis with kernels. Neurocomputing 67, 357-362 (2005) 
6. He, X., Niyogi, P.: Locality preserving projections. In: Thrun, S., Saul, L., Schölkopf, B. (eds.) Advances in Neural Information Processing Systems 16. MIT Press, Cambridge (2004)

7. Asuncion, A., Newman, D.: UCI machine learning repository (2007)

8. Guyon, I., Gunn, S., Ben-Hur, A., Dror, G.: Result analysis of the nips 2003 feature selection challenge. In: Advances in Neural Information Processing Systems, pp. 545-552 (2004)

9. Kanji, G.K.: 100 Statistical Tests. Sage Publications Ltd. (1999) 